


\title{
VARIAÇÃO TEMPORAL DO ESTOQUE DE CARBONO E VOLUME DE MADEIRA EM UM FRAGMENTO DE CERRADO sensu stricto
}

Natielle Gomes Cordeiro', Kelly Marianne Guimarães Pereira², Marcela de Castro Nunes Santos Terra ${ }^{3}$, José Márcio de Mello ${ }^{4}$

${ }^{1}$ Mestranda em Engenharia Florestal pela Universidade Federal de Lavras - UFLA, Lavras, Minas Gerais, Brasil. E-mail: natiellegcordeiro@gmail.com

2 Mestranda em Engenharia Florestal pela Universidade Federal de Lavras - UFLA

${ }^{3}$ Doutora em Engenharia Florestal pela Universidade Federal de Lavras - UFLA

${ }^{4}$ Professor Doutor Adjunto da Universidade Federal de Lavras - UFLA

Recebido em: 22/09/2018 - Aprovado em: 23/11/2018 - Publicado em: 03/12/2018 DOI: 10.18677/EnciBio_2018B76

O Cerrado, considerado como um hotspot mundial, está ameaçado pela expansão de atividades agropecuárias e florestais. Este estudo objetivou avaliar a variação temporal do estoque de carbono e volume de madeira em uma reserva de Cerrado sensu stricto em Minas Gerais, com medições nos anos de 1993, 2000 e 2009. Os dados foram coletados por meio da alocação de unidades amostrais com dimensões de $20 \times 20 \mathrm{~m}$. O critério de inclusão foi a mensuração de todos os indivíduos arbóreos vivos com diâmetro $\geq 5 \mathrm{~cm}$ a altura do peito (DAP), obtido a 1,30 metro em relação ao nível do solo. $\mathrm{Na}$ mesma ocasião, mensurou-se a altura total. Foi calculado o estoque de carbono total (ton) e volume total com casca $\left(\mathrm{m}^{3}\right)$ para cada ano medido, além da quantificação do incremento periódico anual (IPA). As variações temporais entre as medições foram testadas por teste t pareado. Os resultados apontaram estoques de carbono no fuste das árvores variando entre de 11,51 a 34,52 ton.ha ${ }^{-1}$ durante o período considerado, enquanto o volume variou de 41,75 a $116,78 \mathrm{~m}^{3} \cdot \mathrm{ha}^{-1}$. O IPA de carbono foi de 1,40 ton.ano $^{-1}$ para um intervalo de 7 anos e 1,47 ton.ano ${ }^{-1}$ para nove anos. Para o volume, o IPA foi de $4,31 \mathrm{~m}^{3}$.ano ${ }^{-1} \mathrm{e}$ $4,99 \mathrm{~m}^{3}$.ano ${ }^{-1}$. As flutuações representam alterações significativas no estoque de carbono e no volume de madeira de acordo com o teste t. Os ganhos em termos de carbono e volume evidenciam a importância dos fragmentos como sumidouros de carbono, desempenhando assim, papel fundamental na mitigação de mudanças climáticas e antrópicas.

PALAVRAS-CHAVE: Carbono; Inventário Florestal; Savana Brasileira.

\section{TEMPORAL VARIATION OF TREE CARBON STOCK AND WOOD VOLUME IN A CERRADO sensu stricto REMNANT}

\section{ABSTRACT}

Brazilian Cerrado is considered a global hotspot and it is threatened by the expansion of agricultural and forestry activities. This study aimed to evaluate the temporal variation of tree carbon stock and wood volume in a Cerrado sensu stricto remnant, in the Minas Gerais state, with inventories in the years of 1993, 2000 and ENCICLOPÉDIA BIOSFERA, Centro Científico Conhecer - Goiânia, v. 15 n.28; p.931 2018 
2009. Data were collected by systematically laying sample plots of $20 \times 20 \mathrm{~m}$. The sampling criterion was the measurement of all tree individuals with diameter at 1,30 meters of soil $(\mathrm{DBH}) \geq 5 \mathrm{~cm}$. At the same time, the total height was measured. The carbon stock (ton) and total wood volume with bark $\left(\mathrm{m}^{3}\right)$ were calculated for each measurement year. It was calculated the periodic annual increment (PAI). The temporal variations for each area were tested by t paired test. The results showed carbon stocks in the tree trunk varying between 11.51 and 34.52 t.ha $^{-1}$ during the period considered, whereas wood volume varied from 41.75 and $116.78 \mathrm{~m}^{3}$.ha ${ }^{-1}$. The PAl to carbon was 1.40 ton.year $^{-1}$ considering an interval of seven years and 1.47 ton.year ${ }^{-1}$ to nine years. The volume PAl for the intervals was $4.31 \mathrm{~m}^{3}$.year ${ }^{-1}$ and 4.99 $\mathrm{m}^{3}$.year ${ }^{-1}$. The fluctuations in the intervals represent significant changes in the carbon stock and the volume of wood according to the t test. The gains in terms of carbon and volume enhance the importance of these vegetation remnants as carbon sinks, playing a key role in mitigating anthropogenic climate changes.

KEYWORDS: Carbon; Forest inventory; Brazilian Savannna.

\section{INTRODUÇÃO}

O Cerrado se destaca por apresentar grande complexidade paisagística e ecológica, isto é, abriga uma alta diversidade de espécies animais e vegetais, muitas destas apontadas como endêmicas. Este domínio é considerado como um hotspot mundial de diversidade e o segundo maior do país (MYERS et al., 2000). É caracterizado por um gradiente vegetacional que abrange desde formações mais abertas, destacando o Cerrado sensu stricto, até formações florestais mais densas, como o cerradão (REZENDE et al., 2006; SILVA NETO et al., 2016; STRASSBURG et al., 2017).

Nas últimas décadas, o Cerrado vem sofrendo com a expansão de atividades agropecuárias e florestais, que empregadas sem a devida atenção, provocam um alto índice de desmatamento na vegetação, ocasionando perdas da sua estrutura e composição (KLINK; MACHADO, 2005; BARROSO et al., 2017). Nesse sentido, torna-se importante o conhecimento da estrutura da floresta e quantificação dos seus atributos, permitindo a determinação de variáveis imprescindíveis para tomadas de decisão, como o volume de madeira e a quantificação do estoque de carbono presente nas árvores. Tais predições podem ser consideradas, por exemplo, ao se implementar um plano para a recuperação de áreas degradadas, conduzindo a utilização de espécies potenciais, bem como na escolha de áreas prioritárias para conservação (GASPAR et al., 2016).

O conhecimento do estoque de carbono e volume de madeira assim como suas variações temporais são relevantes por permitir a análise do potencial de sequestro de carbono da vegetação. O dióxido de carbono possui uma influência direta no clima e tem sua emissão acentuada com a redução de áreas florestadas, sendo que este fato contribui diretamente para a intensificação do efeito estufa e consequente aquecimento global (REZENDE et al., 2006; RIBEIRO et al., 2009; GASPAR et al., 2014). O sequestro de carbono é, portanto, um importante serviço ambiental prestado pelas áreas verdes, que contribui para a mitigação do aquecimento global e outras mudanças climáticas (PARRON et al., 2015). Assim, o ciclo do carbono está relacionado aos ecossistemas florestais e savânicos, pois estes promovem a assimilação e acumulação do carbono, ressaltando que tais processos são influenciados pelos distúrbios antrópicos e naturais que ocorrem nas florestas (PAIVA et al., 2011). 
No caso específico do Cerrado, são encontrados na literatura estudos quantificando carbono na vegetação. Por exemplo, Scolforo et al. (2016) estimaram o valor de 9,03 ton.ha ${ }^{-1}$ para a parte aérea das árvores em áreas de Cerrado no estado de Minas Gerais, Brasil. Paiva et al. (2011) estimaram 22,83 ton.ha ${ }^{-1}$ para raízes em área de cerrado no Distrito Federal. Morais et al. (2017) encontraram a média de 6,8 Mg.ha-1 para as raízes em Cerrado no município de Olhos d'Água, também em Minas Gerais. Assim, o conhecimento do potencial da vegetação pode ser amparado pela quantificação do estoque de carbono, que é realizada por meio de métodos diretos e indiretos. No que diz respeito à utilização dos métodos diretos, é necessário o abate das árvores mediante o emprego de tecnologias adequadas. Já os métodos indiretos são aplicados nas estimativas do estoque de carbono por meio da utilização de modelos matemáticos, que são obtidos com os dados do inventário florestal, como o diâmetro, altura e a densidade da madeira. Assim também, outras metodologias vêm sendo aplicadas para essa estimativa, por meio de informações de sensoriamento remoto com imagens orbitais (QURESHI et al., 2012).

Tendo em vista o cenário supracitado, este estudo objetivou avaliar a variação temporal do estoque de carbono e volume de madeira em uma reserva de Cerrado sensu stricto situado em Minas Gerais, com três medições nos anos de 1993, 2000 e 2009, por meio da análise de dados dos inventários florestais realizados na área.

\section{MATERIAL E MÉTODOS}

Os dados deste estudo foram provenientes de um fragmento florestal de Cerrado sensu stricto situado em Lavras, Minas Gerais. A área é destinada para pesquisas, portanto trata-se de um fragmento de cerrado bem conservado em que há o controle de ações antrópicas. Conhecida como "Cerradinho da UFLA", esta área é considerada como uma reserva e compreende 2,81 hectares de extensão (PEREIRA et al., 2010). De acordo com a classificação de Köppen, o clima da região é caracterizado como Cwa, com temperatura média anual de $19,3^{\circ} \mathrm{C}$, precipitação anual de $1.530 \mathrm{~mm}$ e altitude de $918 \mathrm{~m}$ (ALVARES et al., 2013).

O inventário florestal consistiu no lançamento de parcelas quadradas de área fixa, cobrindo $100 \%$ da área de estudo, exceto nas bordas onde não foi possível alocar uma parcela de tamanho completo. No primeiro levantamento, em 1993, foram demarcadas e mensuradas 34 unidades amostrais com dimensões de $20 \times 20 \mathrm{~m}\left(400 \mathrm{~m}^{2}\right)$. Estas parcelas foram remedidas nos intervalos posteriores de 2000 e 2009 (ALMEIDA et al., 2014; MARSARO et al., 2017).

O critério de inclusão para a comunidade foi a mensuração de todos os indivíduos arbóreos vivos com diâmetro $\geq 5 \mathrm{~cm}$, obtido a 1,30 metro em relação ao nível do solo (DAP). Na mesma ocasião, mensurou-se a altura total (MORO; MARTINS, 2011). Para os inventários subsequentes foram registradas as árvores mortas, remedidas as sobreviventes e quantificadas as recrutas, ou seja, aquelas que atingiram o nível de inclusão.

Foram calculados o estoque de carbono do fuste das árvores (ton) e o volume de madeira total com casca $\left(\mathrm{m}^{3}\right)$, de acordo com as Equações $1 \mathrm{e} 2$, respectivamente. Posteriormente, obteve-se o estoque de carbono e o volume por parcela, por hectare e total, para cada ano de medição com a utilização de equações ajustadas para a vegetação do Cerrado sensu stricto na região da bacia do Rio Grande (SCOLFORO et al., 2008). 
$\ln (C)=-10,8346535319+1,9968275846 * \ln (\mathrm{DAP})+0,90535240 * \ln (\mathrm{Ht})$

$\ln (\mathrm{VT})=-9,7157262192+2,3511009017^{*} \ln (\mathrm{DAP})+0,50556006 * \ln (\mathrm{Ht})$

Em que:

C: Estoque de carbono (tonelada);

VT: Volume total com casca $\left(\mathrm{m}^{3}\right)$;

DAP: Diâmetro a 1,30 metro ao nível do solo $(\mathrm{cm})$;

$\mathrm{Ht}$ : Altura total da árvore $(\mathrm{m})$.

Por fim, os dados de carbono e volume provenientes das parcelas lançadas na área estudada foram processados de forma a se obter o parâmetro das características mensuradas (estoque de carbono e volume) em cada ano de medição. Subsequentemente determinou-se 0 incremento periódico anual (IPA) destas características para a área de estudo. A variação temporal de carbono e volume de madeira entre as parcelas dentro de cada medição foram testadas por meio de teste t pareado (ZAR, 2010).

\section{RESULTADOS E DISCUSSÃO}

A partir dos levantamentos florestais realizados no fragmento, obtiveram-se os parâmetros diâmetro médio, máximo e mínimo, a altura média, máxima e mínima para cada período, assim como o número de indivíduos mensurados por hectare. Ao avaliar os parâmetros DAP médio e altura média para a reserva, pode-se inferir que os mesmos obtiveram um crescimento progressivo para os anos de medições. Para o intervalo entre 1993 e 2000 houve um aumento do número de indivíduos passando de 2117 para 2492. No entanto, ao analisarmos o intervalo entre 2000 e 2009, notase que não houve um crescimento na quantidade de indivíduos e sim um decréscimo, o qual pode estar associado com a dinâmica da comunidade (Tabela 1).

TABELA 1 - Caracterização dendrométrica e número de indivíduos da reserva de Cerrado sensu stricto

\begin{tabular}{cccccccc}
\hline \multirow{2}{*}{ Monitoramento } & \multicolumn{7}{c}{ Estatísticas } \\
\cline { 2 - 8 } & $\mathbf{D A P}_{\text {min }}$ & $\overline{\mathbf{D A P}}$ & $\mathbf{D A P}_{\text {máx }}$ & $\mathbf{H t}_{\text {min }}$ & $\overline{\mathbf{H t}}$ & $\mathbf{H t}_{\text {máx }}$ & $\mathbf{N}$ \\
\hline $\mathbf{1 9 9 3}$ & 1,12 & 5,66 & 29,63 & 1,00 & 3,93 & 9,30 & 2117 \\
\hline $\mathbf{2 0 0 0}$ & 1,11 & 6,36 & 33,75 & 1,00 & 5,04 & 52,00 & 2492 \\
$\mathbf{2 0 0 9}$ & 1,12 & 7,73 & 42,75 & 1,00 & 5,65 & 18,00 & 2331 \\
\hline
\end{tabular}

$\mathrm{N}=$ número de indivíduos por hectare; $\overline{\mathrm{DAP}}=$ é o diâmetro médio das árvores em centímetros; $\mathrm{DAP}_{\min }=$ diâmetro mínimo $(\mathrm{cm})$; $\mathrm{DAP}_{\text {máx }}=$ diâmetro máximo $(\mathrm{cm}) ; \overline{\mathrm{Ht}}=$ altura média das árvores em metros; $\mathrm{Ht}_{\mathrm{min}}=$ altura total mínima $(\mathrm{m}) ; \mathrm{Ht}_{\mathrm{máx}}=$ altura total máxima $(\mathrm{m})$.

Segundo Santana et al. (2013) é notável uma relação entre o número de indivíduos por hectare e o estoque de carbono de uma dada fitofisionomia. No entanto, o que se constatou nos resultados obtidos para a área de estudo é a não ocorrência desta proporcionalidade, visto que, apesar do maior número de indivíduos para o ano de 2000, o maior estoque de carbono está presente na medição de 2009. Este fato pode ser explicado ao se ponderar que o estoque de carbono está relacionado com as dimensões dos indivíduos arbóreos avaliados, isto 
é, o crescimento das variáveis dendrométricas, altura e DAP, as quais influem diretamente no estoque de carbono, pois sua determinação é oriunda de equações que consideram estas características.

O estoque de carbono para a área apresentou uma variação média de 11,51 a 34,52 ton.ha h $^{-1}$ (Tabela 2), entre os períodos avaliados. Os valores encontrados são superiores quando comparados com o estudo realizado por Schwieder et al. (2018) em três áreas do Cerrado nos estados de Goiás e Mato Grosso, que apresentou um intervalo de carbono de 3,66 a 5,47 ton. ha ${ }^{-1}$. No entanto, ao comparar com o estudo realizado por Ribeiro et al. (2017) no Cerrado sensu stricto em Minas Gerais, em que se quantificou um estoque de carbono de 22,39 ton.ha ${ }^{-1}$, nota-se que as médias de carbono estão bem próximas. Nesse sentido, o estoque de carbono encontra-se dentro do intervalo esperado para o bioma, ressaltando que as variações na quantificação da característica estão possivelmente atreladas a fitofisionomia e ao estado de conservação da área (SANTANA et al., 2013). Ao analisar formações florestais e savânicas no centro do Brasil, Silva et al. (2013) discorreram que o estoque de carbono possui relação intrínseca com o ecossistema de origem, encontrando que ambientes florestais apresentam um armazenamento de carbono quatro vezes maior que ambientes abertos.

Em relação ao estoque volumétrico, assim como o estoque de carbono, obteve-se um aumento ao longo do tempo (Tabela 2). Ponderando os anos de medições para a área, é notável uma variação no volume médio de madeira entre 41,75 a 116,78 $\mathrm{m}^{3}$.ha ${ }^{-1}$. Sanquetta et al. (2018) ao analisarem a dinâmica do Cerrado brasileiro entre os anos de 1990 a 2015, registraram um volume variando de 4,71 a $5,86 \mathrm{~m}^{3} \cdot \mathrm{ha}^{-1}$. Considerando a mesma vegetação avaliada nos fragmentos deste estudo, Meira Junior et al. (2016) encontraram um volume total de madeira de 14,66 $\mathrm{m}^{3}$.ha ${ }^{-1}$. Os autores depreendem que o volume está intimamente atrelado com a diversidade funcional e que a partir desta relação é possível se inferir sobre os serviços ecossistêmicos gerados.

Devido à heterogeneidade inerente à vegetação nativa, em decorrência de suas idiossincrasias, espera-se que o coeficiente de variação seja alto (PÉLLICO NETTO; BRENA, 1997; CALEGARI et al., 2010). Contudo, ao comparar os valores obtidos para o fragmento nos três períodos de mensuração, observa-se que a área apresentou relativamente baixos valores, provavelmente por ser tratar de uma vegetação em estágio avançado de sucessão e por não ocorrer em uma área com grandes mudanças de fatores ambientais (Tabela 2).

TABELA 2 - Valores de carbono (ton) e volume de madeira $\left(\mathrm{m}^{3}\right)$ para 34 unidades amostrais mensuradas no fragmento com vegetação de Cerrado sensu stricto

\begin{tabular}{cccccc}
\hline \multirow{2}{*}{ Ano } & \multirow{2}{*}{ Variável } & \multicolumn{4}{c}{ Estatísticas } \\
\cline { 3 - 6 } & & Média/parcela & Média/ha & Total & CV (\%) \\
\hline \multirow{2}{*}{1993} & Carbono (ton) & 0,46 & 11,51 & 15,66 & 34,83 \\
\cline { 2 - 6 } & Volume (m $\left.{ }^{3}\right)$ & 1,67 & 41,75 & 56,78 & 34,81 \\
\hline \multirow{2}{*}{2000} & Carbono (ton) & 0,85 & 21,32 & 28,99 & 33,17 \\
\cline { 2 - 6 } & Volume $\left(m^{3}\right)$ & 2,88 & 71,89 & 97,78 & 30,91 \\
\hline \multirow{2}{*}{2009} & Carbono (ton) & 1,38 & 34,52 & 46,95 & 38,13 \\
\cline { 2 - 6 } & Volume (m $\left.{ }^{3}\right)$ & 4,67 & 116,78 & 158,82 & 36,69 \\
\hline
\end{tabular}

$\overline{\mathrm{CV}}=$ coeficiente de variação (\%). 
A quantificação do estoque de carbono para o fragmento permitiu inferir que houve um aumento nos valores dessa variável entre os períodos de mensuração. Por meio do teste t pareado se observou que as diferenças entre as médias de estoque de carbono nos anos de medição para a área foram significativas. Verificase na Tabela 3 que o aumento da variável é expressivo para a formação vegetal, representando o quanto o fragmento tem papel importante no sequestro de carbono, o que também pode ser atribuído à capacidade do bioma em termos de regeneração e crescimento (LOPES; MIOLA, 2010).

TABELA 3 - Teste t pareado para o estoque de carbono entre as parcelas avaliadas no Cerrado sensu stricto

\section{Estoque de Carbono - Teste t pareado}

\begin{tabular}{cc}
\hline Período & P-valor \\
\hline $\mathbf{1 9 9 3 - 2 0 0 0}$ & $5,74 \mathrm{E}-15^{\star}$ \\
\hline $\mathbf{2 0 0 0 - 2 0 0 9}$ & $7,45 \mathrm{E}-13^{*}$ \\
\hline
\end{tabular}

*: significativo a 5\%.

Por meio do teste t pareado foi possível afirmar que o aumento de volume de madeira para a reserva foi significativo, considerando que o incremento na variável de interesse mostrou o potencial do bioma em acumular biomassa quando não exposto a maiores distúrbios. Tal fato pode ser explicado devido a característica de interesse, o volume, ser dado em função da altura e do DAP, estabelecendo assim, uma correlação positiva. Com isso, os resultados obtidos (Tabela 4) comprovaram um aumento progressivo das variáveis dendrométricas durante os anos de mensuração, o que influenciou no acréscimo do estoque volumétrico (MIGUEL et al., 2015).

TABELA 4 - Teste t pareado para o volume de madeira entre as parcelas avaliadas no Cerrado sensu stricto.

Volume de madeira - Teste t pareado

\begin{tabular}{cc}
\hline Período & P-valor \\
\hline $\mathbf{1 9 9 3 - 2 0 0 0}$ & $7,85 \mathrm{E}-15^{*}$ \\
\hline $\mathbf{2 0 0 0 - 2 0 0 9}$ & $6,30 \mathrm{E}-13^{*}$ \\
\hline
\end{tabular}

*: significativo a $5 \%$.

Ao avaliar o incremento periódico anual, considerando os dois intervalos existentes entre as mensurações, constatou-se um aumento para as características estoque de carbono e volume de madeira. Deve se ressaltar que, o intervalo de tempo entre os anos de 1993 e 2000 compreendeu uma diferença de sete anos, e para o período de 2000 a 2009, nove anos, o que possivelmente propiciou um aumento no crescimento e desenvolvimento da floresta. A importância dos incrementos está na evidência da capacidade do fragmento em estocar carbono. Mediante os resultados deste estudo, pode-se inferir que o Cerrado em bom estado de conservação consegue aumentar seu estoque de carbono em aproximadamente 1,5 ton.ha ${ }^{-1}$.ano ${ }^{-1}$. Ademais, vale ressaltar que para este estudo o estoque de ENCICLOPÉDIA BIOSFERA, Centro Científico Conhecer - Goiânia, v. 15 n.28; p.936 2018 
carbono foi determinado mediante a mensuração do fuste até $3 \mathrm{~cm}$, não incluindo galhos e folhas, cabendo assim destacar a importância do ecossistema para o ambiente (Tabela 5).

TABELA 5 - Incremento nas características de interesse nos períodos de mensuração entre 1993 e 2009

\begin{tabular}{ccc} 
Períodos & Características & IPA \\
\hline \multirow{2}{*}{$\mathbf{1 9 9 3 - 2 0 0 0}$} & Carbono (ton/ha/ano) & 1,40 \\
\cline { 2 - 3 } & Volume $\left(\mathrm{m}^{3} / \mathrm{ha} / \mathrm{ano}\right)$ & 4,31 \\
\hline \multirow{2}{*}{$\mathbf{2 0 0 0 - 2 0 0 9}$} & Carbono (ton/ha/ano) & 1,47 \\
\cline { 2 - 3 } & Volume (m³/ha/ano) & 4,99
\end{tabular}

$\mathrm{IPA}=$ incremento periódico anual por hectare.

O conhecimento da biomassa arbórea permite predizer sobre a composição e estrutura da floresta, pois auxilia nas tomadas de decisão (COLGAN et al., 2014). Com isso, o aumento progressivo do estoque de carbono e volume de madeira na área indicou crescimento dos indivíduos arbóreos e recrutamento de novos na amostragem. Esse padrão refletiu o estado de conservação da área, a qual possui controle sobre as ações antrópicas, sendo que possivelmente são bem preservadas e livre de incêndios. Ao mesmo tempo, em áreas de Cerrado, é importante ressaltar que o acúmulo de biomassa eleva o risco de incêndios, principalmente na estação seca (SILVA et al., 2011).

A área apresentou ganho significativo em estoque de carbono e volume de madeira, o que significa que esta atua como sumidouro de carbono, desempenhando, portanto, importante serviço ambiental e apresentando potencial para conservação, principalmente diante das mudanças climáticas antrópicas (IPCC, 2012; SOUZA; SOARES, 2013). A determinação e conhecimento do estoque de carbono existente em uma floresta nativa permite inferir quanto aos serviços ambientais prestados, assim como fornece informações precisas para as tomadas de decisão quanto aos procedimentos a serem adotados para recuperação de áreas degradadas e consequente redução do efeito estufa (SANTOS et al., 2016).

\section{CONCLUSÃO}

A análise de inventários sucessivos, monitoramento efetuado pelas 3 medições, mostrou que o fragmento de Cerrado sensu stricto localizado em Minas Gerais apresentou ganho significativo de carbono e volume total de madeira ao longo do tempo. Houve um aumento aproximado de $33 \%$ no estoque de carbono e de $36 \%$ no volume da primeira para a última medição. Nesse contexto, os ganhos em termos de carbono e volume de madeira evidenciam a importância do fragmento como sumidouro de carbono, desempenhando assim, papel fundamental na mitigação de mudanças climáticas antrópicas. 


\section{AGRADECIMENTOS}

Os autores agradecem à Universidade Federal de Lavras (UFLA) pela estrutura e disponibilidade dos recursos necessários para desenvolvimento da pesquisa, assim, como a Coordenação de Aperfeiçoamento de Pessoal de Nível Superior (CAPES) pelo auxílio financeiro.

\section{REFERÊNCIAS}

ALMEIDA, A. Q. de; MELLO, A. A. de; DÓRIA NETO, A. L.; FERRAZ, R. C. Relações empíricas entre características dendrométricas da Caatinga Brasileira e dados TM Landsat 5. Pesquisa Agropecuaria Brasileira, v. 49, n. 4, p. 306-315, 2014. Disponível em: <http://dx.doi.org/10.1590/S0100-204X2014000400009>. doi: 10.1590/S0100-204X2014000400009

ALVARES, C. A.; STAPE, J. L.; SENTELHAS, P. S.; GONÇALVES, J. L. de M.; SPAROVEK, G. Köppen's climate classification map for Brazil. Meteorologische Zeitschrift, v. 22, n. 6, p. 711-728, 2013. Disponível em: < http://dx.doi.org/10.1127/0941-2948/2013/0507>. doi: 10.1127/0941-2948/2013/0507 BARROSO, A. G.; SANO, E. E.; FREITAS, D. M. de. Identificação de desmatamentos no Cerrado utilizando técnicas de diferença de imagens e índice de queimada por diferença normalizada. Revista Brasileira de Cartografia, v. 69, n. 7, p. 1397-1409, 2017. Disponível em: < http://www.rbc.Isie.unb.br/index.php/rbc/article/view/1906/1218>. Acesso em: 23 ago. 2018.

CALEGARI, L.; MARTINS, S. V.; GLERIANI, J. N.; SILVA, E.; BUSATO, L. C. Análise da dinâmica de fragmentos florestais no município de Carandaí, MG, para fins de restauração florestal. Revista Árvore, v. 34, n. 5, p. 871-880, 2010. Disponível em: < http://dx.doi.org/10.1590/S0100-67622010000500012>. doi: 10.1590/S0100-67622010000500012

COLGAN, M. S.; SWEMMER, T.; ASNER, G. P. Structural relationships between form factor, wood density, and biomass in African savanna woodlands. Trees, v. 28, p. 91-102, 2014. Disponível em: <http://dx.doi.org/10.1007/s00468-013-0932-7> doi: 10.1007/s00468-013-0932-7

GASPAR, R. de O.; CASTRO, R. V. O.; PELOSO, R. V. D.; SOUZA, F. C. de; MARTINS, S. V. Análise fitossociológica e do estoque de carbono no estrato arbóreo de um fragmento de floresta estacional semidecidual. Ciencia Florestal, v. 24, n. 2 , p. 313-324, 2014. Disponível em: <http://dx.doi.org/10.5902/1980509814569>. doi: 10.5902/1980509814569

GASPAR, R. O.; LUSTOSA JUNIOR, I. N.; RODRIGUES, M. I.; ARAÚJO, J. B. C. N.; LOBÃO, M. S. Dendrocronologia na análise do crescimento em diâmetro, volume, biomassa e dióxido de carbono no Cerrado. Nativa, v. 4, n. 1, p. 48-52, 2016. Disponível em: <http://dx.doi.org/10.14583/2318-7670.v04n01a10>. doi: 10.14583/2318-7670.v04n01a10

IPCC. Managing the Risks of Extreme Events and Disasters to Advance Climate Change Adaptation. 1 ed. Cambridge, UK, and New York, NY, USA: Cambridge University Press, 2012.

KLINK, C. A.; MACHADO, R. B. A conservação do Cerrado brasileiro. Megadiversidade, v. 1, n. 1, p. 147-155, 2005. Disponível em: < https://www.agencia.cnptia.embrapa.br/recursos/Texto_Adicional_ConservacaolDxNOKMLsupY.pdf>. Acesso em: 23 ago. 2018. 
LOPES, R. B.; MIOLA, D. T. B. Sequestro de carbono em diferentes fitofisionomias do Cerrado. SynThesis Revista Digital FAPAM, v. 2, n. 2, p. 127-143, 2010. Disponível em:

http://periodicos.fapam.edu.br/index.php/synthesis/article/view/40/37>. Acesso em: 23 ago. 2018.

MARSARO, C. B.; NAKAJIMA, N. Y.; MACHADO, S. do A.; MELO, L. O.; RUSCHEL, A. R. et al. Eficiência relativa de duas configurações de parcelas de área fixa para inventário do potencial madeireiro na Amazônia Oriental. Nativa, v. 5, n. esp., p. 574-580, 2017. Disponível em: <http://dx.doi.org/10.5935/2318-7670.v05nespa17>. doi: 10.5935/2318-7670.v05nespa17

MEIRA JUNIOR, M. S.; IMAÑA ENCINAS, J.; PINTO, J. R. R.; MOTA, S. da L. L. Functional diversity influence in forest wood stock: a study of the Brazilian Savanna.

Bioscience Journal, v. 32, n. 6, p. 1619-1631, 2016. Disponível em: <http://dx.doi.org/10.14393/BJ-v32n1a2016-33189>. doi: 10.14393/BJ-v32n1a201633189

MIGUEL, E. P.; REZENDE, A. V.; LEAL, F. A.; MATRICARDI, E. A. P.; VAlE, A. T. do. et al. Redes neurais artificiais para a modelagem do volume de madeira e biomassa do cerradão com dados de satélite. Pesquisa Agropecuaria Brasileira, v. 50, n. 9, p. 829-839, 2015. Disponível em: <http://dx.doi.org/10.1590/S0100204X2015000900012>. doi: 10.1590/S0100-204X2015000900012

MORAIS, V. A.; SANTOS, C. S.; MELLO, J. M.; DAVID, H. C.; ARAÚJO, E. J. G. et al. Spatial and vertical distribution of litter and bellowground carbon in a Brazilian Cerrado vegetation. Cerne, v. 23, n. 1, p. 43-52, 2017. Disponível em: < http://dx.doi.org/10.1590/01047760201723012247>.

doi: 10.1590/01047760201723012247

MORO, M. F.; MARTINS, F. R. Métodos de levantamento do componente arbóreo arbustivo. In: FELFILI, J. M.; EISENLOHR, P. V.; MELO, M. M. R. F.; ANDRADE, L. A.; MEIRA NETO, J. A. A. (Org.). Fitossociologia no Brasil: Métodos e Estudos de Caso - Volume 1. Viçosa: Editora UFV, 2011. p. 174-212.

MYERS, N.; MITTERMEIER, R. A.; MITTERMEIER, C. G.; FONSECA, G. A. B. da; KENT, J. Biodiversity hotspots for conservation priorities. Nature, v. 403, n. February, p. 853-858, 2000. Disponível em: <https://doi.org/10.1038/35002501>. doi: $10.1038 / 35002501$

PAIVA, A. O.; REZENDE, A. V.; PEREIRA, R. S. Estoque de Carbono em Cerrado sensu stricto do Distrito Federal. Revista Árvore, v. 35, n. 3, p. 527-538, 2011. Disponível em: <http://dx.doi.org/10.1590/S0100-67622011000300015>. doi: 10.1590/S0100-67622011000300015

PARRON, L. M.; GARCIA, J. R.; RACHWAL, M. F. G.; FRANCHINE, J. C.; FRANCISCON, L. et al. Avaliação de serviços ambientais no âmbito do projeto ServiAmbi. In: PARRON, L. M.; GARCIA, J. R.; OLVEIRA, E. B. de; BROWN, G. G.; PRADO, R. B. (Org.). Serviços Ambientais em Sistemas Agrícolas e Florestais do Bioma Mata Atlântica. Brasília: EMBRAPA, 2015. p. 36-46. 2015.

PEREIRA, I. M.; van den BERG. E.; PINTO, L. V. de A.; HIGUCHI, P.; CARVALHO, D. A. de. Avaliação e proposta de conectividade dos fragmentos remanescentes no campus da Universidade Federal de Lavras, Minas Gerais. CERNE, vol.16, n.3, p.305-321, 2010. Disponível em: <http://dx.doi.org/10.1590/S010477602010000300007>. doi: 10.1590/S0104-77602010000300007

QURESHI, A.; PARIVA; BADOLA, R.; HUSSAIN, S. A. A review of protocols used for assessment of carbon stock in forested landscapes. Environmental Science and 
Policy, v. 16, p. 81-89, 2012. Disponível em: <http://dx.doi.org/10.1016/j.envsci.2011.11.001>. doi: 10.1016/j.envsci.2011.11.001 REZENDE, A. V.; VALE, A. T. do; SANQUETTA, C. R.; FIGUEIREDO FILHO, A.; FELFILLI, J. M. Comparação de modelos matemáticos para estimativa do volume, biomassa e estoque de carbono da vegetação lenhosa de um cerrado sensu stricto em Brasília, DF. Scientia Forestalis, v. 71, n. Agosto, p. 65-76, 2006. Disponível em: <http://www.ipef.br/publicacoes/scientia/nr71/cap07.pdf>. Acessado em: 23 ago. 2018.

RIBEIRO, S. C.; JACOVINE, L. A. G.; SOARES, C. P. B.; MARTINS, S. V.; SOUZA, A. L. de. et al. Quantificação de biomassa e estimativa de estoque de carbono em uma floresta madura no município de Viçosa, Minas Gerais. Revista Árvore, v. 33, n. 5, p. 917-926, 2009. Disponível em: <http://dx.doi.org/10.1590/S010067622009000500014>. doi: 10.1590/S0100-67622009000500014

RIBEIRO, S. C.; JACOVINE, L. A. G.; TORRES, C. M. M. E.; SOUZA, A. L. de. Influence of interspecific variation on tree carbon stock of a Brazilian Cerrado. Revista Árvore, v. 41, n. 5, p. 1-11, 2017. Disponível em: <http://dx .doi.org/10.1590/1806-90882017000500006>. doi: 10.1590/180690882017000500006

SANQUETTA, C. R.; CORTE, A. P. D.; PElissARI, A. R.; TOMÉ, M.; MAAS, G. C. $B$. et al. Dinâmica em superfície, volume, biomassa e carbono nas Florestas Nativas Brasileiras: 1990-2015. BIOFIX Scientific Journal, v. 3, n. 1, p. 193-198, 2018. Disponível em: <http://dx.doi.org/10.5380/biofix.v3i1.58513>. doi: 10.5380/biofix.v3i1.58513

SANTANA, O. A.; IMAÑA ENCINAS, J., INÁCIO, E. dos S. B.; AMORIM, L. B. de; VILA VERDE, J. L. J. Relação entre o índice de avermelhamento do solo e o estoque de carbono na biomassa aperea da vegetação de Cerrado. Ciencia Florestal, v. 23, n. 4, p. 783-794, 2013. Disponível em: <http://dx.doi.org/10.5902/1980509812362>. doi: 10.5902/1980509812362

SANTOS, R. C. dos; CASTRO, R. V. O.; CARNEIRO, A. de C. O.; CASTRO, A. F. N. M.; PIMENTA, A. S. et al. Estoques de volume, biomassa e carbono na madeira de espécies da Caatinga em Caicó, RN. Pesquisa Florestal Brasileira, v. 36, n. 85, p. 1-7, 2016. Disponível em: <https://doi.org/10.4336/2016.pfb.36.85.772>. doi: 10.4336/2016.pfb.36.85.772

SCHWIEDER, M.; LEITÃO, P. J.; PINTO, J. R. R.; TEIXEIRA, A. M. C.; PETRONI, F. et al. Landsat phenological metrics and their relation to aboveground carbon in the Brazilian Savanna. Carbon Balance and Management, v. 13, n. 7, p. 1-15, 2018. Disponível em: <https://doi.org/10.1186/s13021-018-0097-1>. doi: 10.1186/s13021018-0097-1

SCOLFORO, J. R. S.; OLIVEIRA, A. D; ACERBI JÚNIOR, F. W. Inventário florestal de Minas Gerais: Equações de Volume, Peso de Materia Seca, e Carbono para diferentes fitosionomias da Flora Nativa. 1 ed. Lavras: UFLA, 2008.

SCOLFORO, H. F.; SCOLFORO, J. R. S.; MELLO, C. R.; MELLO, J. M.; FERRAZ FILHO, A. C. Spatial Distribution of Aboveground Carbon Stock of the Arboreal Vegetation in Brazilian Biomes of Savanna, Atlantic Forest and Semi-Arid Woodland. Plos One, v. 10, n. 6, p. 1-20, 2015. Disponível em: <https://doi.org/10.1371/journal.pone.0128781>. doi:10.1371/journal.pone.0128781 SOUZA, A. L.; SOARES, C. P. B. Florestas Nativas - Estrutura, dinâmica e manejo. 1a ed. Viçosa: Editora UFV, 2013. 
SILVA, D. M. Da; LAIOLA, P. de P.; ROSATTI, N. B.; SILVA, I. A.; CIANCIARUSO, $M$. V. et al. Os efeitos dos regimes de fogo sobre a vegetação de cerrado no Parque Nacional das Emas, GO: considerações para a conservação da diversidade. Biodiversidade Brasileira, v. 1, n. 2, p. 26-39, 2011. Disponível em: < http://www.icmbio.gov.br/revistaeletronica/index.php/BioBR/article/view/136/102>. Acesso em: 23 ago. 2018.

SILVA, L. C. R.; HOFFMANN, W. A., ROSSATTO, D. R.; HARIDASAN, M.; FRANCO, A. C. et al. Can savannas become forests? A coupled analysis of nutrient stocks and fire thresholds in central Brazil. Plant and Soil, v. 373, p. 829-842, 2013. Disponível em: <http://dx.doi.org/10.1007/s11104-013-1822-x>. doi: 10.1007/s11104013-1822-x

SILVA NETO, V. L.; OLIVEIRA, A. L. de; FERREIRA, R. Q. de S.; SOUZA, P. B. de; VIOLA, M. R. Fitossociologia e distribuição diamétrica de uma área de Cerrado sensu stricto, Dueré - TO. Revista de Ciências Ambientais, v. 10, n. 1, p. 91-106, 2016. Disponível em: <http://dx.doi.org/10.18316/1981-8858.16.24>. doi: 10.18316/1981-8858.16.24

STRASSBURG, B. B. N.; BROOKS, T.; FELTRAN-BARBIERI, R.; IRIBARREN, A.; CROUZEILLES, R. et al. Moment of truth for the Cerrado hotspot. Nature Ecology and Evolution, v. 1, n. 0099, p. 1-3, 2017. Disponível em: < http://dx.doi.org/10.1038/s41559-017-0099>. doi: 10.1038/s41559-017-0099

ZAR, J. H. Biostatistical Analysis. 5a ed. Prentice-Hall/Pearson, Upper Saddle River - NJ: Prentice-Hall, 2010. 